\title{
Barcelona
}

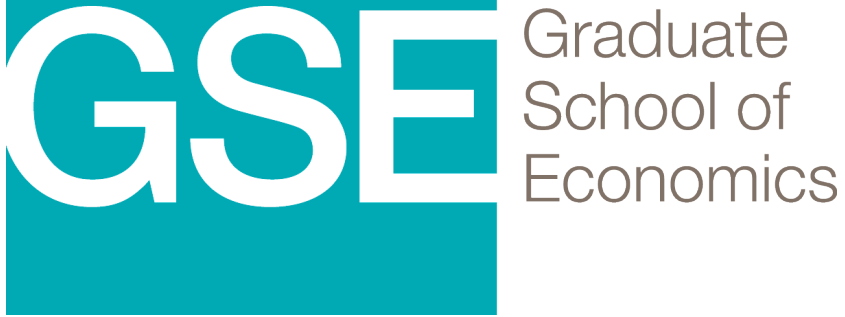

\section{Balancing the Power to Appoint Officers}

Salvador Barberà

Danilo Coelho

This version October 2015

(May 2013)

Barcelona GSE Working Paper Series

Working Paper $n^{\circ} 696$ 


\title{
Balancing the Power to Appoint Officers
}

\section{Salvador Barberà (MOVE, UAB and Barcelona GSE) and Danilo Coelho (IPEA)*}

\author{
October 1, 2015
}

\begin{abstract}
Rules of $\mathrm{k}$ names are frequently used methods to appoint individuals to office. They are two-stage procedures where a first set of agents, the proposers, select $\mathrm{k}$ individuals from an initial set of candidates, and then another agent, the chooser, appoints one among those $\mathrm{k}$ in the list. In practice, the list of $\mathrm{k}$ names is often arrived at by letting each of the proposers screen the proposed candidates by voting for $\mathrm{v}$ of them and then choose those $\mathrm{k}$ with the highest support. We then speak of v-rules of $\mathrm{k}$ names. Our main purpose in this paper is to study how different choices of the parameters $\mathrm{v}$ and $\mathrm{k}$ affect the balance of power between the proposers and the choosers. From a positive point of view, we analyze a strategic game where the proposers interact to determine what list of candidates to submit. From a normative point of view, we study the impact of the choice of parameters $\mathrm{v}$ and $\mathrm{k}$ upon the distribution of power among the proposers and the chooser, and we discuss how to eventually balance it.
\end{abstract}

Key Words: Voting rules; Constitutional Design; Strong Nash equilibrium; Rule of $k$ Names. JEL classification: D02, D71, D72.

*salvador.barbera@uab.cat and danilo.coelho@ipea.gov.br. The authors gratefully acknowledge support from the Spanish Ministry of Science and Innovation through grant "Consolidated Group-C" ECO2008-04756 and FEDER, from the Generalitat de Catalunya, Departament d'Universitats, Recerca i Societat de la Informació through the Distinció per a la Promoció de la Recerca Universitària and grant SGR2009-0419. Salvador Barberà acknowledges financial support from the Spanish Ministry of Economy and Competitiveness, through the Severo Ochoa Programme for Centres of Excellence in R\&D (SEV-2011-0075). Danilo Coelho acknowledges the financial support from the Brazilian Ministry of Science Technology and Innovation, through CNPq Research Productivity Scholarship. We thank Miguel Ballester, Alexandre Carvalho, Anke Gerber, Matthew Jackson, David Jimenez, Oscar Nupia and Carmelo Rodríguez-Álvarez for useful comments. 


\section{Introduction}

Appointing people to office is one of the main ways how the powerful exert their influence in society. But the ability of any authority to appoint officers is often limited by the existence of other "de iure" or "de facto" powers.

In this paper we study a class of methods that allow several agents to share the power to appoint. We call them rules of $k$ names, and they work as follows. The set of deciders is divided into two groups: the proposers and the chooser. Proposers consider the set of all candidates to a position and screen $k$ of them. Then, the chooser picks the appointee out of these $k$ names.

Indeed, rules of $k$ names can vary, depending on the composition of the set of proposers, on the value of $k$, and also on the voting procedure adopted to form a list of $k$ candidates.

Here we focus in a specific family of rules of $k$ names that are used in many practical cases. This family adopts the following procedure to form the list of $k$ names: each proposer votes for $v$ candidates, and then the $k$ most voted candidates get into the list. Though one can think of other methods to select the $k$ names, the ones we consider are simple and frequently used. We call the resulting systems $v$-rules of $k$ names.

Rules of that form have been used in the past and are still very much used in the present. They seem particularly fit to give partial decision power to different parties that are interested in the workings of an institution, and want to have a voice when selecting its officers. Historically, rules of $k$ names were used within the Roman Church since the early middle ages, when secular rulers tried to control the appointment of bishops, while the clergy would rather decide on its leaders. And similar rules are still used to share the power between Rome and the local congregations. At present, in many countries (US, Argentina, Brazil, Canada, Chile, Haiti, Mexico, Spain, Turkey etc.), the seats in appellate courts are filled through the use of rules of $k$ names. This is the case, for example, of the commission-selection, political appointment method to select judges to the state supreme courts of several US states, sometimes referred to as merit selection or the Missouri Plan. They are also used in several countries (Brazil and Turkey) to appoint the Rectors of public universities.

It is clear that the size $k$ of the list to be submitted has an important effect on the distribution of power between the proposers and the chooser. In the extreme case where the proposers must submit the whole list of candidates, all power goes to the chooser. 
In the opposite extreme case where $k=1$, it is the chooser who has no room left, and all the power stays in the hands of the proposers ${ }^{1}$. We shall focus in the intermediate, non-degenerate cases where both the proposers and the chooser have influence on the final decision, and study how the values of $v$ and $k$ affect the actual distribution of power between the two parties. Specifically, we shall be interested in the kind of ex ante evaluation that a designer could make of different $v$-rules of $k$ names.

The methods that are actually used in practice differ in the values of these definitional parameters: the size of the list is not always the same, nor its relationship with the number of votes that each proposer is allowed. There are instances where in order to participate in the choice of $k$ candidates, each voter is allowed to submit $k$ names. The rule used to elect Irish bishops or prosecutor-general in most Brazilian states are of this sort, with $k=v=3$. Yet, in most cases we know, each proposer is asked to submit a vote for $v$ candidates, with $v$ less than $k$. This is the case, for example, when choosing public university rectors in Brazil $(k=3 ; v=1)$, members of Chile's courts of justice $(k=3 ; v=2)$ or Chile's Supreme Court $(k=5 ; v=3)$.

In order to understand what is the actual power of each party under any given rule, we first study the strategic behavior of different agents operating under it. This positive point of view leads us to analyze a strategic game of interaction between the different proposers, when determining what list to submit. Much of our work is geared to identify the resulting equilibria for different values of $v$ and $k$. Armed with this understanding of equilibria, and the power distribution that they imply, we can then turn to more normative questions. Can we balance the power among the parties? If so, what choices of parameters will be appropriate?

The paper is organized as follows. In the next section we provide a summary of our findings in preceding papers on rules of $k$ names, and then discuss several related papers. Section 3 provides the general setup and a discussion of the strategic issues that arise. In view of their complexity, Section 4 concentrates on the analysis of power balance within the limits of a specific but still rich model that focuses in the case where agents are polarized. Conclusions follow in Section 5, and proofs appear in the Appendix.

\footnotetext{
${ }^{1}$ In that case, however, there is still room for analysis, to determine what candidate would arise, depending on $v$ and on the preferences of proposers.
} 


\section{Literature review}

There does not seem to be a body of literature specifically devoted to study appointment rules with their checks and balances. Of course there exist many voting rules that can be adapted to this specific purpose, and rules of $k$ names can also be used for other purposes. But we think it is useful to focus in the case of appointments, and to pay special attention to methods that especially fit that purpose.

We have studied rules of $k$ names in two preceding papers. Barberà and Coelho (2006) focuses on the properties of set valued screening rules, the first-stage ingredient of rules of $k$ names. Specifically, we show that several screening rules that are used in practice, including the ones we study in the present paper, may not always select weak Condorcet sets of candidates when these exist and voters act straightforwardly ${ }^{2}$. This could be a cause of instability if the proposers were not strategic, but we argue that this is an additional reason to consider the performance of screening rules in connection with the overall game that is generated by $v$-rules of $k$ names.

Barberà and Coelho (2010) is a more substantial paper, concentrating in the characterization of the strong Nash equilibria of different games that one can associate with the use of rules of $k$ names. In particular, we distinguish between two models. In one, to be used again in the present paper, we concentrate in the strategic interactions among the proposers. In a second model, we allow for the chooser to also join coalitions with proposers. An important conclusion of our study was to establish that the exact form of the internal rules used by the proposers is not always essential. What really matters is whether these rules are or are not majoritarian. A rule is majoritarian if it is the case that agents in any majority of proposers always have actions at their disposal allowing them, if properly coordinated, to impose the full list of $k$ names, whatever other agents $\mathrm{do}^{3}$. For each one of the two games we consider, we show that the characterization of the sets of strong Nash equilibrium outcomes is the same for all majoritarian rules of $k$ names, irrespectively of the details in the internal rules used by proposers. A drawback of our analysis is that strong Nash equilibria may fail to exist. However, we were able to prove existence for the case where the agents' preferences are single peaked, and perform

\footnotetext{
${ }^{2} \mathrm{~A}$ weak Condorcet set (Gehrlein, 1985) is one such that no candidate inside in it can be majority defeated by any outside candidate.

${ }^{3}$ Notice that this does not mean that a majoritarian group will always use that potential.
} 
some comparative statics on the value of $k$.

The distinction between those rules that are majoritarian and those that are not is important in our 2010 paper that we just summarized, and it also provides motivation for our study of $v$-rules of $k$ names in the present one. This is because $v$-rules of $k$ names are always majoritarian when $v=k$, while not necessarily if $v<k$, as in many rules used in real life. The strong Nash equilibria of non-majoritarian rules require a more detailed analysis, since the role of minority proposers becomes then more complex and interesting. Concentrating on $v$-rules allows us to understand their workings in a context that covers many more rules than those we considered in our preceding work.

We now turn attention to related papers dealing with procedures that are similar but not exactly equal to our rules of $k$ names. An exception is a recent paper by de Clippel, Eliaz and Knight (2014), showing that the rule of $k$ names where $k$ is equal to $\frac{c+1}{2}$ ( $c$ is the number of candidates) implements what they call the Veto-Rank mechanism where two agents simultaneously veto $\frac{c+1}{2}$ candidates and the winner is the one with the minimal sum of ranks among those who have not been vetoed. This result reinforces the idea that our proposed rules, in general, are appropriate methods to achieve a compromise solution among different participants in a collective decision.

Holzman and Moulin (2013) and Alon et al. (2011) concentrate on what they call nomination rules, leading to the choice of a fixed number of candidates, when the candidates are also the voters. Even if different from ours in many respects, these papers also show that being specific on the nature of the choice to be made, and on the actors being involved, allows to sharpen the positive and normative questions to be asked about appointment rules. This is also the case for a variety of sequential methods, where different agents play different roles, as voters or vetoers. See, for example, Mueller's (1978) voting by veto, Moulin's (1982) successive elimination procedures, or Stevens (1966) and Brams and Merril's (1986) final-offer arbitrage procedures.

Our normative analysis of rules of $k$ names will be based in expected utility calculations, similar in spirit to those made by Rae (1969), Curtis (1972), Badger (1972), Coelho (2004), Barberà and Jackson (2004) and Attanasi et al. (2010), when comparing different voting schemes. However, these papers concentrate on societies that face dichotomous choices, while we allow sets of alternatives of any size.

Although all of these related methods are of theoretical interest, it is fair to say that 
rules of $k$ names stand out as being the most widely used in practice, among those defined in a similar vein.

Finally, let us mention Ertemel, Kutlu and Sanver (2015), Sertel and Sanver (2004), Polborn and Messner (2007), Moulin (1982) and Gardner (1977) as some of the papers that characterize the strong Nash equilibrium outcomes of different voting games.

\section{The setup}

In this section we formally define rules of $k$ names and the games they induce. We observe that, in addition to other structural features, like the number of proposers, the number of candidates and the size $k$ of proposed candidates, a full specification of a rule of $k$ names also requires to define the screening rules by which the proposers decide what names go into the list. In principle, this method could remain unspecified, or be rather complicated. But in actual practice simple and specific screening rules are used, and we concentrate on them. Proposers are allowed to vote for a number $\mathrm{v}$ of candidates, and then the $k$ most voted ones are selected (with a tie break if needed). These votes will typically be cast as the result of strategic calculations that may involve the cooperative coordination among players.

Let $\boldsymbol{C}$ be the finite set of candidates and $c$ be its cardinality. For any $h<c, \boldsymbol{C}_{h}$ denotes the family of subsets of $\boldsymbol{C}$ with cardinality $h$. Let $\boldsymbol{N}=\{1, \ldots, n\}$ be the finite set of proposers. The set of agents is $\boldsymbol{A}=\mathbf{N} \cup\{$ chooser $\}$, where chooser is interpreted as an individual not in $\boldsymbol{N}$. Let $\boldsymbol{P}$ be the set of all strict orders ${ }^{4}$ on $\boldsymbol{C}$. Elements in $\boldsymbol{P}$ are denoted by $\succ_{i}, \succ_{j}, \ldots$

Societies, or preferences profiles, are elements of $\boldsymbol{P}^{n+1}$, denoted as $\left(\succ_{1}, \succ_{2}, \ldots, \succ_{n}\right.$ , $\left.\succ_{\text {chooser }}\right)^{5}$. The first $n$ components are interpreted to be the preferences of the proposers and the last component stands for the preferences of the chooser.

In addition to stand for the preferences of agents, orders of the set of candidates will also be used to break ties among alternatives, as we shall be later. Given an order $\succ$, and

\footnotetext{
${ }^{4}$ Transitive: For all $x, y, z \in \mathbf{C}:(x \succ y$ and $y \succ z)$ implies that $x \succ z$.

Asymmetric: For all $x, y \in \mathbf{C}: x \succ y$ implies that $\neg(y \succ x)$.

Irreflexive: For all $x \in \mathbf{C}, \neg(x \succ x)$.

Complete: For all $x, y \in \mathbf{C}: x \neq y$ implies that $(y \succ x$ or $x \succ y$ ).

${ }^{5}$ The superscript $n$ refers to the $n$-fold Cartesian product of sets.
} 
any subset of candidates $\boldsymbol{B} \subset \boldsymbol{C}$, we denote by $\alpha(\mathbf{B}, \succ)$ the best candidate in $\mathbf{B}$ according to $\succ$.

We now define $v$-rules of $k$ names. Both $k$ and $v$ are integers, $k \leq c$ is the size of the set of candidates that the proposers must submit and $v \leq k$ is the number of candidates that each proposer can support. The parameter $v$ must be $\frac{k}{n} \leq v \leq k{ }^{6}$

Definition 1 Given any n-tuple $\left(\mathbf{B}_{1}, \ldots, \mathbf{B}_{n}\right)$ belonging to $\boldsymbol{C}_{v}^{n}$, of sets of size $v$, the score of a candidate $x \in \boldsymbol{C}$ at $\left(\boldsymbol{B}_{1}, \ldots, \boldsymbol{B}_{n}\right)$ is the number of $\boldsymbol{B}_{i}$ 's containing $x, s\left(x, \boldsymbol{B}_{1}, \ldots, \boldsymbol{B}_{n}\right)=$ $\#\left\{\boldsymbol{B}_{i} \mid x \in \boldsymbol{B}_{i}\right\}$. A set $\boldsymbol{T}$ is most voted in $\left(\boldsymbol{B}_{1}, \ldots, \boldsymbol{B}_{n}\right)$ if for all $x \in \boldsymbol{T}$ and $y \in \boldsymbol{C} \backslash \boldsymbol{T}$, $s\left(x, \boldsymbol{B}_{1}, \ldots, \boldsymbol{B}_{n}\right) \geq s\left(y, \boldsymbol{B}_{1}, \ldots, \boldsymbol{B}_{n}\right)$. A v-screening rule of $k$ names is a function $g: \boldsymbol{C}_{v}^{n} \rightarrow \boldsymbol{C}_{k}$ that selects a set $\boldsymbol{T}$ of $k$ most voted candidates for each $n$-tuple of sets of size $v$.

Definition 2 A v-rule of $k$ names is a function $f: \boldsymbol{C}_{v}^{n} \times \boldsymbol{P} \rightarrow \boldsymbol{C}$ defined so that $f\left(\boldsymbol{B}_{1}, \ldots, \boldsymbol{B}_{n}, \succ\right.$ )$=\alpha\left(g\left(\left(\boldsymbol{B}_{1}, \ldots, \boldsymbol{B}_{n}\right)\right), \succ\right)$, for some $v$-screening rule of $k$ names $g$.

Notice that our definition of a set of most voted candidates allows for some candidates in $\boldsymbol{T}$ and some outside of $\boldsymbol{T}$ to get the same number of votes. This is because, in our setting, there may be cases where several candidates get the same number $h$ of votes, the set of those getting more than $h$ votes is smaller than $k$ and the set of those getting at least $h$ votes is larger than $k$. In these cases, the screening rule must "break the tie" between these candidates who just got $h$ votes, and select enough of them to complete a set of size $k$. From now on, we will assume that our "tie breaking rules" are given by an order of candidates, and that this order is either fixed, or coincides with the preferences of some predetermined agent ${ }^{7}$.

An important part of our work will consist in analyzing the type of strategic interactions that may arise among the proposers, as a function of their preferences and those of the chooser. We model these interactions as a normal form game with complete information, and concentrate our analysis on the study of its strong Nash equilibria.

Definition 3 (Barberà and Coelho, 2010) Given $k \in\{1,2, \ldots, c\}$ and $v \in\{1,2, \ldots, k\}, a$ $v$-screening rule for $k$ names $g: \boldsymbol{C}_{v}^{n} \rightarrow \boldsymbol{C}_{k}$ and a preference profile $\left(\succ_{1}, \succ_{2}, \ldots, \succ_{n}, \succ_{\text {chooser }}\right.$

\footnotetext{
${ }^{6}$ The lower bound guarantees that there exists a voting configuration where all the $k$ selected candidates receive at least one vote, while the upper bound is always respected in practice.

${ }^{7}$ For example, when $k=3$ and $\boldsymbol{C}=(x, y, z, w)$, if x,y receive 3 votes and the remaining two candidates $z, w$ receive the same number of votes (say 2 , one or none), the proposed set will be formed by $x, y$ and the highest ranked among $z$ and $w$ according to the tie breaking rule.
} 
) $\in \boldsymbol{P}^{n+1}$, the Constrained Chooser Game is the simultaneous game with complete information where each player $i \in \boldsymbol{N}$ chooses a strategy $\boldsymbol{B}_{i} \in \boldsymbol{C}_{v}$. Given $\left(\boldsymbol{B}_{1}, \ldots, \boldsymbol{B}_{n}\right) \in \boldsymbol{C}_{v}^{n}$, $g\left(\left(\boldsymbol{B}_{1}, \ldots, \boldsymbol{B}_{n}\right)\right)$ is the chosen list with $k$ names and the winning candidate is $\alpha\left(g\left(\left(\boldsymbol{B}_{1}, \ldots, \boldsymbol{B}_{n}\right)\right), \succ_{\text {chooser }}\right)$.

In the Constrained Chooser Game, the chooser's strategy set is restricted to a single element. In that sense, we could say that she is not an active player. Specifically, we take it that the chooser will simply select the candidate that is best for her among those that she will be presented with. Thus, the chooser's preferences determine the game's outcome function, and will have an impact on the equilibrium play of the proposers. But, in the spirit of subgame perfection, and given the sequential form of our rules, we exclude the possibility that the chooser may select a candidate that is not her best in the list she is presented with.

We choose to analyze the set of strong Nash equilibria of this game. This is consistent with the idea that proposers have complete information about their preferences and those of the chooser, and that they must find ways to cooperate among themselves, in order to come up with a favorable list.

Definition 4 Given $k \in\{1,2, \ldots, c\}$ and $v \in\{1,2, \ldots, k\}$, a $v$-screening rule for $k$ names $g: \boldsymbol{C}_{v}^{n} \longrightarrow \boldsymbol{C}_{k}$ and a preference profile $\succ \equiv\left\{\succ_{i}\right\}_{i \in \boldsymbol{N} \cup\{\text { chooser }\}} \in \boldsymbol{P}^{n+1}$, a joint strategy $\left(\boldsymbol{B}_{1}, \ldots, \boldsymbol{B}_{n}\right) \in \boldsymbol{C}_{v}^{n}$ is a pure strong Nash equilibrium of the Constrained Chooser Game if and only if, given any coalition $\boldsymbol{G} \subseteq \boldsymbol{N}$, there is no $\left(\boldsymbol{B}_{1}^{\prime}, \ldots, \boldsymbol{B}_{n}^{\prime}\right) \in \boldsymbol{C}_{v}^{n}$ with $\boldsymbol{B}_{j}^{\prime}=\boldsymbol{B}_{j}$ for every $j \in \boldsymbol{N} \backslash \boldsymbol{G}$ such that $\alpha\left(g\left(\left(\boldsymbol{B}_{1}^{\prime}, \ldots, \boldsymbol{B}_{n}^{\prime}\right)\right), \succ_{\text {chooser }}\right) \succ_{i} \alpha\left(g\left(\left(\boldsymbol{B}_{1}, \ldots, \boldsymbol{B}_{n}\right)\right), \succ_{\text {chooser }}\right.$ ) for each $i \in \mathbf{G}$.

Finding the equilibria of the games generated under different $v$-rules of $k$ names is thus a necessary step, prior to the choice of normatively attractive values for $v$ and $k$. In our search for equilibria and their characterizations, we will face a number of difficulties, that eventually lead us to concentrate on a simple model. But in order to give the reader a feeling of the interesting problems that arise, let us present the following two examples.

Example 1 There are five candidates $\{c 1, c 2, c 3, c 4, c 5\}$ and eleven proposers. Each proposer is allowed to vote for one candidate $(v=1)$ and a list will be formed with the names of the three most voted candidates $(k=3)$, with ties being broken according to the order 
$c 1 \succ c 3 \succ c 4 \succ c 5 \succ c 2$. The type (preferences) and the number of agents are given in the following table.

\section{Preference Profile}

1 type 1 proposer 7 type 2 proposers 3 type 3 proposers Chooser

$\begin{array}{llll}c 1 & c 3 & c 2 & c 1 \\ c 3 & c 2 & c 3 & c 2 \\ c 4 & c 5 & c 4 & c 3 \\ c 5 & c 5 & c 4 \\ c 2 & c 1 & c 1 & c 5\end{array}$

We shall argue, in what follows, that $c 2$ can be the outcome induced from the strong Nash equilibrium play of the proposers when the chooser always picks his best candidate in the list.

Consider the following strategy profile that sustains c2 as a strong Nash equilibrium outcome: the seven type 2 proposers cast four votes for $c 3$ and three votes for $c 4$. The only one type 1 proposer casts a vote for 1 , while the three type 3 proposers cast three votes for $c 2$. Thus, the selected list is $\{c 3, c 2, c 4\}$ and $c 2$ is the winning candidate.

The argument behind this equilibrium is quite clear. Type 3's go ahead in support of c2, and then the type 2's have to prevent c1 from becoming the outcome by "wasting" their remaining votes in support of $c 4$.

But there is another, maybe more interesting equilibrium. Notice that any coalition with at least three proposers can impose at least one candidate in the list, and that the chooser and the three proposers of type 3 prefer $c 2$ to $c 3$. In spite of this, candidate $c 3$ can also be sustained as a strong Nash equilibrium outcome! To verify it, consider the following strategy profile: the seven type 2 proposers cast three votes for 33 , two votes for 4 , one vote for $c 1$ and one vote for $c 5$. Type 1 proposer casts a vote for $c 1$, while the three type 3 proposers cast two votes for $c 5$ and one for $c 4$. So, $33, c 5$ and $c 4$ will have three votes each, while $c 1$ only two. Thus, the selected list is $\{c 3, c 5, c 4\}$ and $c 3$ is the winning candidate. The reader can check that no coalition of voters can profitably deviate.

Now, here is a intuition for this equilibrium, where the two proposers of type 2 cleverly distribute their votes in order to prevent the type 3's from being able to select c2, even if they all vote for it. Voters of type 2 ensure that candidate 33 , their favorite, is among the proposed ones, by casting three votes in its favor. They also give enough support to 
candidate 1 so that, along with the vote of type 1, c1 is still not chosen, but would be as soon as candidates with two votes enter the list. Then, since $c 1$ has two votes, proposers of type 3 cannot vote for their favorite, c2, because if they all spent their votes on $c 2$, which would make c2 eligible, then some alternative with two votes would come in, and in this case it would be c1, which they hate but is the chooser's best. Given that they cannot get $c 2$, they then concentrate, in alliance with type 2 people, in getting $c 4$ and c5 into the list, both above their worse alternative $c 1$, in order to at least get their second alternative. Thus, the presence of the type 1 proposer voting for $c 1$ leads types 2 and 3 into a sort of race: if one of them uses the most rewarding strategy in one of the two equilibria, the other must concede. If both used their most rewarding strategies, then $c 1$, that they both hate, would come out?

In this example, we can observe several types of strategic behavior on the side of agents. The richness of the example also leads to the existence of several equilibria among which it is hard to choose. Multiplicity of equilibria adds to the difficulty of characterizing any of them. Hence, even if the steps to be taken toward any specific choice of optimal rules are quite clear, we cannot expect simple, general explicit solutions. This is why we shall eventually simplify the setting where we work.

Our second example is also clarifying. The choice of $v$ and $k$ has an impact on the balance between the satisfaction of the chooser and that of the proposers. But our following example shows that this impact is complex: without any further restrictions, the effects of $k$ and $v$ on the agents' payoffs are not monotonic.

Example 2 There are four candidates $c 2, c 1, c 3$ and $c 4$, and three proposers. Each proposer votes for one candidate and the list has the names of the two most voted candidates $(v=1, k=2)$, with a tie breaking rule when needed: $c 3 \succ c 4 \succ c 1 \succ c 2$.

\footnotetext{
${ }^{8}$ Notice that the same outcome c3 could also be sustained by other strategy profiles. For instance, if the seven type 2 proposers cast two votes for $\mathrm{c} 3, \mathrm{c} 4$, and $\mathrm{c} 5$ each and one vote for $\mathrm{c} 1$, given the truthful vote for $\mathrm{c} 1$ of the type 1 proposer, the type 3 proposers can do no better than cast one vote each for $\mathrm{c} 3$, $\mathrm{c} 4$, and $\mathrm{c} 5$ in order to ensure that $\mathrm{c} 1$ does not make the shortlist on the tie-break. Thus, it is possible to get c3 as a strong Nash equilibrium outcome even if type 2 proposers don't ensure that c 3 is on the shortlist. We thank a referee for pointing this out.
} 
Preference Profile

Proposer 1 Proposer 2 Proposer 3 Chooser

$\begin{array}{cccc}c 1 & c 2 & c 2 & c 1 \\ c 4 & c 3 & c 3 & c 2 \\ c 3 & c 4 & c 4 & c 3 \\ c 2 & c 1 & c 1 & c 4\end{array}$

Candidate $c 3$ is the unique strong Nash equilibrium outcome under $v=1$ and $k=2$. Here is an intuition for this result: notice that candidate c2 cannot be a strong equilibrium outcome, because as long as proposer 1 votes for c1, proposers 2 and 3 cannot get $c 2$ to be the outcome, even if they can force $c 2$ to be in the list. Short of that, proposers 2 and 3 coordinate their actions so that one of them votes for $c 3$ and the other for $c 4$. If 1 persists in voting for $c 1$, this creates a tie between the three candidates that is solved in favor of $c 3$ and $c 4$, out of which the chooser selects $c 3$. If 1 votes for c 3 instead, the same outcome ensues. And all other actions by any combination for agents would lead some of them to outcomes that would be worse than c3 for some of them. Hence, $c 3$ is the unique strong Nash equilibrium under $(v, k)=(1,2)$. The case $(v, k)=(1,1)$ is simple and leads to the election of $c 2$.

The table below presents the set of strong Nash equilibrium for different values of $v$ and $k$.

Set of strong Nash equilibrium outcomes

$$
\begin{array}{lll}
k=1 & v=1 & \{c 2\} \\
k=2 & v=1 & \{c 3\} \\
k=2 & v=2 & \{c 2\} \\
k=3 & v=1 & \{c 1\} \\
k=3 & v=2 & \{c 2\}
\end{array}
$$

Notice that, with $v$ fixed at 1 , the chooser prefers $k=1$ to when $k=2$. This is quite surprising, since $k=1$ means that the chooser has no power at all! Notice also that she prefers $k=3$ to $k=2$ when $v=1$. On the other hand, for $k=2$, the chooser prefers the higher value $v=2$ to that of $v=1$. However, for $k=3$, she prefers the lower value $v=1$ to that of $v=2$.

In spite of the difficulties that these examples point to, we can still provide some partial 
characterization of strong Nash equilibria for the general case, and a statement regarding their connection across different values of $v$ and $k$. We now present these results, before further specializing our model in the next section. Although a bit involved, our conditions shed light on the type of coalitions one should focus in, and they are also useful to search for equilibria in specific applications.

Notice that any $v$-screening rule of $k$ names endows each group of proposers with some power to determine what candidates are to be included in the list submitted to the chooser. The following definitions and results apply for any given $v$-rule of $k$ names and any subset $\boldsymbol{X}$ of $\boldsymbol{C}$ with size smaller than or equal to $k .^{9}$

Definition 5 Let $q_{k}^{v}(\boldsymbol{X})$ be the minimum integer $\widehat{q}$ such that, for any coalition $\mathbf{G}$ of voters with size at least as large as $\widehat{q}$, agents in $\boldsymbol{G}$ can vote in such a way that all elements in $\boldsymbol{X}$ are included in the list, for any vote of the proposers in $\boldsymbol{N} \backslash \boldsymbol{G}$. That is, $q_{k}^{v}(\boldsymbol{X})$ is computed in such a way that any coalition of that size or larger can always guarantee itself the inclusion of $\boldsymbol{X}$ in the list, if its members coordinate their votes.

Remark 1 The values of $q_{k}^{v}(\cdot)$ evolve monotonically with those of $k$ and $v$. For any $\boldsymbol{C}$ and $v<v^{\prime}<k<k^{\prime}<c$, we have that:

1. $q_{k}^{v}(\boldsymbol{X}) \geq q_{k}^{v^{\prime}}(\boldsymbol{X})$ for any $\boldsymbol{X} \in \boldsymbol{C}_{k}$;

2. $q_{k^{\prime}}^{v}\left(\boldsymbol{X}^{\prime}\right) \geq q_{k}^{v}(\boldsymbol{X})$ for any $\boldsymbol{X} \in \boldsymbol{C}_{k}$ and $\boldsymbol{X}^{\prime} \in\left\{\boldsymbol{Y} \in \boldsymbol{C}_{k^{\prime}} \mid \boldsymbol{X} \subset \boldsymbol{Y}\right\}$;

3. $q_{k}^{v^{\prime}}(\{x\}) \geq q_{k}^{v}(\{x\})$ for any $x \in \boldsymbol{C}$

4. $q_{k}^{v}(\{x\}) \geq q_{k^{\prime}}^{v}(\{x\})$ for any $x \in \boldsymbol{C}$.

Remark 1 tells us that, for every $\boldsymbol{X} \in \boldsymbol{C}_{k}$ and $x \in \boldsymbol{C}, q_{k}^{v}(\boldsymbol{X})$ is increasing in $k$ and decreasing in $v$, while $q_{k}^{v}(\{x\})$ is decreasing in $k$ and increasing in $v$. Thus, an increase in $k$ or a decrease in $v$ alters the distribution of power among the proposers in the following ways: (a) it affects non-positively the cardinality of the set of possible coalitions of players that are able to impose all the names in the list and (b) it affects non-negatively the

\footnotetext{
${ }^{9}$ Notice that definitions 5 and 6 are closely linked to that of effectivity functions studied by Peleg (1984), Moulin and Peleg (1982), Abdou and Keiding (1991) and Sertel and Sanver (2004), among others. The concept of effectivity refers to the ability of agents to ensure that the outcome of a given rule belongs to a target set, and admits several variants.
} 
cardinality of the set of possible coalitions of players that are able to impose at least one name in the list. Notice that (a) implies that some strong Nash equilibrium outcomes under $(v, k)$ may not be a strong Nash equilibrium under $(v-1, k)$ or under $(v, k+1)$. Notice also that (b) implies that the chooser's best candidate in the list may become an equilibrium outcome under $(v-1, k)$ or under $(v, k+1)$ in spite of not being an equilibrium outcome under $(v, k)$.

These $q_{k}^{v}$ values may differ (but not too much) for sets of the same size, depending on the names of the alternatives that they include, because the tie breaking rule treats candidates asymmetrically. Hence, we may also define some absolute bounds that work for any set. In particular, we'll use those bounds that apply for singletons or for sets of size $k$, since they are the ones that will help in characterizing equilibria.

Definition 6 Let $q_{1}^{v} \equiv \operatorname{Max}_{y \in \boldsymbol{C}}\left\{q_{k}^{v}(\{y\})\right\}$ and $q_{k}^{v} \equiv \operatorname{Max}_{\boldsymbol{Y} \in \boldsymbol{C}_{k}}\left\{q_{k}^{v}(\boldsymbol{Y})\right\} .{ }^{10}$

We are now ready to provide a necessary condition that must be satisfied by any strong Nash equilibrium outcomes for the Constrained Chooser Game, whatever the preferences of agents might be. In addition to its intrinsic interest, the result will be later used in our analysis of the polarized proposers' case.

Proposition 1 If candidate $x$ is a strong Nash equilibrium outcome of the Constrained Chooser Game, then it satisfies the following four conditions

C1: It is among the chooser's $(c-k+1)$-top candidates.

C2: If $y \neq x$ is among the chooser's $(c-k+1)$-top candidates then $\#\left\{i \in \mathbf{N} \mid y \succ_{i} x\right\}<$ $q_{k}^{v}(\boldsymbol{Y})$ for any $\boldsymbol{Y} \in \boldsymbol{C}_{k}$ such that $y$ is the chooser's best candidate in $\boldsymbol{Y}$.

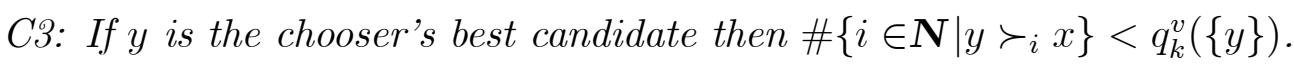

C4: If $y$ is the chooser's best candidate and also ranked above $x$ by the tie breaking criterion, then $\#\left\{i \in \mathbf{N} \mid x \succ_{i} y\right\} \geq q_{k}^{v} .^{11}$

\footnotetext{
${ }^{10}$ We can actually compute these bounds explicitly, as follows: $q_{k}^{v}=\left\lceil\frac{k n}{(k+v)}\right\rceil+\mathcal{I}\left(\left\lfloor\frac{v\left\lceil\frac{k n}{(k+v)}\right\rceil}{k}\right\rfloor \leq n-\right.$ $\left.\left\lceil\frac{k n}{(k+v)}\right\rceil\right)$ and $q_{1}^{v}=\left\lceil\frac{v n}{(k+v)}\right\rceil+\mathcal{I}\left(\frac{v n}{(k+v)}=\left\lceil\frac{v n}{(k+v)}\right\rceil\right)$, where $\mathcal{I}$ denotes the indicator function that takes value 1 if the expression in brackets is true, and 0 otherwise, $\lfloor x\rfloor$ is the largest integer not greater than $x$ and $\lceil x\rceil$ is the smallest integer greater or equal than $x$.

${ }^{11}$ In Appendix B, we show how Proposition 1 can be useful to locate an equilibrium outcome. We also give an example where the set of strong Nash equilibrium outcomes is empty.
} 
In the general case where all preferences are allowed, no conclusive statement can be reached regarding the gains for the chooser from parameter changes, as shown by Example 2. The difficulty to make definite statements under a universal domain of preferences is compounded by the possibility that, when changing parameters, one of them may guarantee existence of equilibria but not the other. In spite of these added difficulties, we can prove the following result that holds for the universal domain of preferences:

Proposition 2 If the chooser's best candidate is a strong Nash equilibrium outcome of the Constrained Chooser Game under a v'-rule for $k^{\prime}$ names then it is also a strong Nash equilibrium outcome of the Constrained Chooser Game under any other v-rule for $k$ names with $v \leq v^{\prime}$ and $k \geq k^{\prime}$, provided that both screening rules use the same tie breaking criterion.

\section{Choosing among v-rules of $\mathrm{k}$ names under polar- ized opinions.}

In this section we study how the choice of the parameters $v$ and $k$ can help to satisfy different normative properties, like the equalization of the power among different groups of participants in an appointment. Before engaging in that analysis, we present a family of societies to which we restrict attention. Examples 1 and 2 in the preceding section suggest that we cannot expect to develop a full general characterization of the equilibrium outcomes when proposers are sufficiently heterogeneous. This is a serious obstacle for the kind of analysis we propose later in that section, and the reason why we focus in cases where the opinions of voters are polarized. We shall argue that this particular case provides significant insights, while preserving the simplicity that we need to proceed.

\subsection{The polarized opinions model.}

From now on, we limit attention to societies where the number of proposers is odd. Actually, we can take this as a natural condition that a designer would want to impose from the onset, when fixing the appointment rule to be used under changing circumstances. We say that a voting problem is polarized if it satisfies the following characteristics.

1. (A1). The set $\boldsymbol{G}$ of proposers is partitioned into two groups $\boldsymbol{G}_{1}$ and $\boldsymbol{G}_{2}$. 
2. (A2). All proposers in $\boldsymbol{G}_{1}$ share the same preferences over the set of candidates.

3. (A3). All proposers in $\boldsymbol{G}_{2}$ share with the chooser the same preferences over the set of candidates.

4. (A4). The chooser's preferences are used to break ties when this is needed to complete the list of $k$ names.

We think that this simple model captures much of what matters when applying rules of $k$ names. It allows for the chooser to have discrepancies with some of the proposers, while agreeing with some others. Hence, it reflects well the tension between two segments of society whose views on the ruler's judgement do not agree. In fact, there are instances where this alignment between the chooser and some proposers may come from the very nature of the institutions that use the rule. For example, in some of the US states where judges of the State Supreme Court are appointed according to the Missouri Plan, the merit committee that acts as the set of proposers is formed by lawyers appointed by the Bar Association and also by non-lawyers appointed by the Governor, who are likely to be aligned with the latter's views.

An advantage of the Constrained Chooser Game associated to our Polarized Opinions Model is that it always has an easy to describe and unique strong Nash equilibrium, as stated in the following proposition.

Proposition 3 Consider the Polarized Opinions Model and any v-rule of $k$ names. There exists a unique strong Nash equilibrium outcome for the associated Constrained Chooser Game. If $\# \boldsymbol{G}_{1} \geq q_{k}^{v}$, then the strong Nash equilibrium outcome is the best candidate of the proposers in $\boldsymbol{G}_{1}$ out of the chooser's (c-k+1)-top candidates. Otherwise, the strong Nash equilibrium outcome is the chooser's best candidate.

Proposition 3 states that if the size of $\boldsymbol{G}_{1}$ is large enough, so that this group is able to impose all names in the list $\left(\# \boldsymbol{G}_{1} \geq q_{k}^{v}\right)$ then the equilibrium outcome is the best candidate for this group out of the $c-k+1$-top candidates of the chooser. Agents in $\boldsymbol{G}_{1}$ can obtain this outcome by imposing a list containing the candidate they want to be chosen, along with $k-1$ candidates from the bottom of the chooser's preferences. In the rest of the cases, $\boldsymbol{G}_{2}$ is able to impose the top candidate of the tie breaking criterion. 
Since the tie breaking criterion coincides with the preferences of the chooser, her best candidate will be the equilibrium outcome.

We can be even more specific for the special case where the partition of $\mathrm{G}$ is degenerate and all proposers have identical preferences.

Corollary 1 If all proposers are homogeneous, i.e. $\boldsymbol{G}_{1}=\mathbf{N}$ and $\boldsymbol{G}_{2}=\phi$, then the unique Strong Nash Equilibrium outcome is the best candidate of the proposers out of the chooser's $(c-k+1)$-top candidates.

Example 3 Let $\boldsymbol{C}=\{c 1, c 2, c 3, c 4, c 5\}$ and let $\mathbf{N}=\{1,2,3\}$. Suppose that each proposer votes for two candidates and the three most voted candidates form the list, with a tie breaking rule when needed: $c 1 \succ c 2 \succ c 3 \succ c 4 \succ c 5$. The preferences of the chooser and the proposers are as follows:

\section{Preference Profile}

Proposer 1 Proposer 2 Proposer 3 Chooser

$\begin{array}{llll}c 5 & c 5 & c 1 & c 1 \\ c 4 & c 4 & c 2 & c 2 \\ c 3 & c 3 & c 3 & c 3 \\ c 1 & c 1 & c 4 & c 4 \\ c 2 & c 2 & c 5 & c 5\end{array}$

Notice that all the assumptions A1-A4 of the Polarized Opinions Model are satisfied, and the values of the parameters are $n=3, \# \mathbf{G}_{1}=2, \# \mathbf{G}_{2}=1, v=2$ and $k=3$. Notice also that $q_{k}^{v}=3$, which implies that $\# \mathbf{G}_{1}<q_{k}^{v}$. Therefore, according to Proposition 3, the equilibrium outcome is the chooser's best candidate. The following strategy profile sustains $c 1$ as a strong Nash equilibrium outcome: Proposer 3 votes for $c 1$ and $c 2$, Proposers 2 and 3 vote for $c 4$ and $c 5$. The list will be formed by $c 1, c 4$ and $c 5$ and $c 1$ will be the winning candidate.

The following two corollaries apply to the case where our $v$-rules of $k$ names are used in societies with polarized opinions. They follow from Proposition 3 and Remark 1.

Corollary 2 Whenever $\widetilde{v}>v^{\prime}$, the chooser cannot be worse off under a $v^{\prime}$-rule for $k$ names than under a $\widetilde{v}$-rule for $k$ names. 
Corollary 3 Whenever $k^{\prime}>\widetilde{k}$, the chooser cannot be worse off under a v-rule for $k^{\prime}$ names than under a $v$-rule for $\widetilde{k}$ names .

As shown by Corollaries 2 and 3 above, in the case of the Polarized Opinions Model, the chooser will always weakly prefer a smaller $v$ and a larger $k$. As for the proposers, and given their polarization, some of them will gain and some will loose from any given parameter change.

\subsection{The ex-ante analysis of different rules}

We will now present the type of calculations that a designer could make in order to determine the utility that the proposers and the chooser may expect to obtain from the use of alternative $v$-rules of $k$ names, in societies with polarized opinions.

We will produce explicit computations under appropriate assumptions, that provide insights on the trade-offs between the choices of $v$ and $k$, and the impact of these choices upon the agents involved in the decision process. Our computations are specific, but the general method to be used, if given alternative data, will become apparent.

We compare the performance of different rules under the assumption that agents are endowed with expected utility functions, and that the designer's objectives involve calculations regarding their expected payoffs, as the preferences of agents change within the bounds of the Polarized Opinions Model.

We will assume here that the designer takes the (negative of the) ranking of the different candidates as a proxy for the utility they derive from their election. In what follows, and for simplicity, $x$ will stand for the equilibrium outcome at each profile. Formally, $u_{i}(x)=-r_{i}$ where $r_{i}=1+\#\left\{z \in \boldsymbol{C} \mid z \succ_{i} x\right\}$. We denote by $u_{\boldsymbol{G}_{1}}(x), u_{\boldsymbol{G}_{2}}(x)$ and $u_{\text {chooser }}(x)$ the utilities of the proposers in groups $\boldsymbol{G}_{1}$ and $\boldsymbol{G}_{2}$ and of the chooser, respectively, given that $x$ is the equilibrium outcome. ${ }^{12}$

Under these simple specifications, each preference order is associated to one utility function, and from now on we use the terms preference profile and utility profile interchangeably.

\footnotetext{
${ }^{12}$ One could argue directly that minimizing the expected ranking of the chosen alternatives is already a natural objective. But we refer to this measure of performance as a utility, in order to emphasize that a planner could attribute alternative objectives to its subjects, and change its optimization criteria accordingly.
} 
If the designer is endowed with a distribution indicating the probability that each profile of preferences is realized, then she is ready to compute the expected utility from choosing each possible value of $k$ and $v$.

For the purpose of illustration, all the results in this section are based on the assumption that the agents' preferences over the set of candidates are the result of two independent random draws from a uniform distribution over the domain of all strict preferences: one to determine the preferences of agents in $\mathbf{G}_{1}$, and another to determine those of the chooser and proposers in $\boldsymbol{G}_{2}$.

Consider the case where $\boldsymbol{G}_{1}$ is large enough such that it is able to impose all names in the list $\left(\# \boldsymbol{G}_{1} \geq q_{k}^{v}\right.$ ). By Proposition 3, the equilibrium outcome is the best candidate for this group out of the $c-k+1$-top candidates of the chooser. Given this characterization of the equilibrium outcome and the assumption of independence between the preferences of the chooser's and of those of $\boldsymbol{G}_{1}$, the random variable $r_{\boldsymbol{G}_{1}}$ has the same distribution as that of the smallest element of a random sample with size $s=c-k+1$ drawn without replacement from a uniformly distributed population $\boldsymbol{D}=\{1,2, \ldots, c\}$ with mean equal to $\frac{(c+1)}{s+1}=\frac{(c+1)}{(c-k+2)} \cdot 13$ And the random variable $r_{\text {chooser }}\left(r_{\text {chooser }} \equiv 1+\#\left\{y \in \boldsymbol{C} \mid y \succ_{\text {chooser }} x\right\}\right)$ has the same distribution as that of a discrete random variable uniformly distributed over $\{1,2, \ldots, c-k+1\}$ with mean equal to $\frac{(c-k+1)+1}{2}$.

Consider now the case where $\boldsymbol{G}_{1}$ is not able to impose all names in the list ( $\# \mathbf{G}_{1}<q_{k}^{v}$ ). By Proposition 3, the equilibrium outcome is the chooser's best candidate. Thus, the random variable $r_{G_{1}}$ is a discrete random variable uniformly distributed over $\{1,2, \ldots, c\}$ with mean equal to $\frac{c+1}{2}$ and $r_{\text {chooser }}$ is a constant equal to 1 .

Proposition 4 Consider the Polarized Opinions Model and any v-rule of $k$ names. The agents' expected utilities are given by the following expressions:

1. If $\# \mathbf{G}_{1} \geq q_{k}^{v}$ then:

$$
\begin{aligned}
& E\left(u_{\boldsymbol{G}_{1}}(x) \mid c, k, v\right)=-\frac{(c+1)}{(c-k+2)} \\
& E\left(u_{\boldsymbol{G}_{2}}(x) \mid c, k, v\right)=E\left(u_{\text {chooser }}(x) \mid c, k, v\right)=-\frac{(c-k+2)}{2}
\end{aligned}
$$

\footnotetext{
${ }^{13}$ For the readers not familiar with order statistics, an introductory text on this topic that has a proof of this formula is available at http://www.math.uah.edu/stat/urn/OrderStatistics.html\#mom1
} 
2. If $\# \mathbf{G}_{1}<q_{k}^{v}$ :

$$
\begin{aligned}
& E\left(u_{\boldsymbol{G}_{1}}(x) \mid c, k, v\right)=-\frac{(k+1)}{2} \\
& E\left(u_{\boldsymbol{G}_{2}}(x) \mid c, k, v\right)=E\left(u_{\text {chooser }}(x) \mid c, k, v\right)=-1
\end{aligned}
$$

Now, given these expected utility formulas, the designer can select a $(v, k)$ satisfying any desirable criteria. Notice that if $\# \boldsymbol{G}_{2} \geq \frac{n+1}{2}$ then $\# \mathbf{G}_{1}<q_{k}^{v}$ and it implies that the agents's expected payoffs are not affected by the parameters $k$ and $v$ of the rule since the chooser's best candidate always win. So, we will only consider the case where $\# \boldsymbol{G}_{1} \geq \frac{n+1}{2}$. We have considered three possible criteria for selection. These criteria will treat the two groups of agents as the two relevant entities participating in the distribution of power. They are related to the notions of egalitarianism, utilitarianism and bargaining, as shown in the following definitions. Yet, alternative criteria could be defined, as we shall comment below.

Definition $7 A$ pair $(v, k)$, such that $k \in\{1, \ldots, c\}, v \in\{1, \ldots, k\}$ and $v \leq k$, is an egalitarian solution if $\left|E\left(u_{\boldsymbol{G}_{1}}(x) \mid c, k, v\right)-E\left(u_{\text {chooser }}(x) \mid c, k, v\right)\right| \leq \mid E\left(u_{\boldsymbol{G}_{1}}(x) \mid c, k^{\prime}, v^{\prime}\right)-$ $E\left(u_{\text {chooser }}(x) \mid c, k^{\prime}, v^{\prime}\right) \mid$ for every $k^{\prime} \in\{1, \ldots, c\}$ and $v^{\prime} \in\left\{1, \ldots, k^{\prime}\right\}$. We denote by $S_{e}$ the set of all values of $(k, v)$ that are egalitarian solutions.

Definition 8 A pair $(v, k)$, such that $k \in\{1, \ldots, c\}, v \in\{1, \ldots, k\}$ and $v \leq k$, is a utilitarian solution if $E\left(u_{\boldsymbol{G}_{1}}(x) \mid c, k, v\right)+E\left(u_{\text {chooser }}(x) \mid c, k, v\right) \geq E\left(u_{\boldsymbol{G}_{1}}(x) \mid c, k^{\prime}, v^{\prime}\right)+E\left(u_{\text {chooser }}(x) \mid c, k^{\prime}, v^{\prime}\right)$ for every $k^{\prime} \in\{1, \ldots, c\}$ and $v^{\prime} \in\left\{1, \ldots, k^{\prime}\right\}$. We denote by $S_{u}$ the set of all values of $(k, v)$ that are utilitarian solutions.

Definition 9 A pair $(v, k)$, such that $k \in\{1, \ldots, c\}, v \in\{1, \ldots, k\}$ and $v \leq k$, is a Nash bargaining solution if $\left(E\left(u_{\boldsymbol{G}_{1}}(x) \mid c, k, v\right)-d\right)\left(E\left(u_{\text {chooser }}(x) \mid c, k, v\right)-d\right) \geq\left(E\left(u_{\boldsymbol{G}_{1}}(x) \mid c, k^{\prime}, v^{\prime}\right)-\right.$ $d)\left(E\left(u_{\text {chooser }}(x) \mid c, k^{\prime}, v^{\prime}\right)-d\right)$ for every $k^{\prime} \in\{1, \ldots, c\}$ and $v^{\prime} \in\left\{1, \ldots, k^{\prime}\right\}$ where $d$, the status quo expected utility for each of the players, is equal to $-\frac{c+1}{2}$. We denote by $S_{\boldsymbol{N}}$ the set of all values of $(k, v)$ that are Nash bargain solutions.

Interestingly, these three criteria lead to the selection of the same values for $(v, k)$, in our case when $\# \boldsymbol{G}_{1} \geq \frac{n+1}{2}$. The reason is simple: the combination of expected utilities 
for the proposers in group $\boldsymbol{G}_{1}$ and for the chooser that we get as $k$ changes, keeping $v$ such that $\# \mathbf{G}_{1} \geq q_{k}^{v}$ (this condition always holds when $v=k$ and $\# \mathbf{G}_{1} \geq \frac{n+1}{2}$ ), constitute a symmetric set. Since the egalitarian and the utilitarian solutions satisfy Nash's axiom of symmetry, and our bargaining problem is symmetric, they both coincide with Nash's solution in this nice case. In fact, they may lead to the choice of one or at most two values of $k$ depending on the number of candidates. At any rate, we can always say that the value of $k$ that balance the power is greater than half the number of candidates. That fact may be a bit disturbing, since in real life we observe the use of small values of $k$. But this is due to the specificity of the polarized case and the complete information assumption, where the majority proposer gets an advantage, that can only be compensated by a relatively larger $k$.

Let us insist that these definitions exploit the fact that agents come in two distinct groups, and treat each group as one of the two references for a division of power. If we wanted to get close to the standard notion of utilitarianism, for example, we would have had to consider the sums of the agents' utilities in each of the groups, and that would give rise to more complex calculations and more controversy regarding the weights that each of the parties should be give. At any rate, if we had to single out one of the three criteria, we think that the egalitarian solution is the one that is more amenable to an analysis of the distribution of power among single agents, rather than between blocks.

The following proposition expresses our preceding remarks more formally and with additional detail.

Proposition 5 Consider the Polarized Opinions Model and $\# \mathbf{G}_{1} \geq \frac{n+1}{2}$. The egalitarian, utilitarian and Nash bargaining solutions of $(v, k)$ coincide. Let $z=c+\frac{5}{2}-\sqrt{2 c+\frac{9}{4}}$. If $z$ is an integer then the value of $k$ in any egalitarian solution must be equal to $z$ or $z-1$, otherwise $k$ is equal to $\lfloor z\rfloor .{ }^{14}$ If $(v, k)$ is an egalitarian solution then $\left(v^{*}=k, k\right)$ is also an egalitarian solution. Full equalization of expected utilities is achieved if and only if $k=c-\sqrt{2 c+2}+2$. The value of the optimal $k$ 's according to any egalitarian, utilitarian and Nash bargaining solution is always greater than or equal to $\frac{c+1}{2}$.

Example 4 Consider the Polarized Opinions Model and let $c=7, n=5$ and $\# \mathbf{G}_{1}=4$. It implies that $z=c+\frac{5}{2}-\sqrt{2 c+\frac{9}{4}}=7+\frac{5}{2}-\sqrt{10+\frac{9}{4}}=5.47$. Thus, by Proposition

\footnotetext{
${ }^{14}\lfloor z\rfloor$ is the largest integer not greater than $z$.
} 
5, if $(v, k)$ is an egalitarian solution then $k=\lfloor z\rfloor=5$. Proposition 5 also tells us that if $k=c-\sqrt{2 c+2}+2=5$ then $(v, k)$ (with $v$ is such that $\# \mathbf{G}_{1} \geq q_{k}^{v}$ ) equalizes the agent's expected utilities. Table 1 shows that only the following pairs of parameters equalize the agents expected utilities: $(v=3, k=5),(v=4, k=5)$ and $(v=5, k=5)$. 
Table 1: Agents' expected utilities for different values of $k$ and $v$ when $c=7, n=5$,

$\# \mathbf{G}_{1}=4$

\begin{tabular}{|c|c|c|c|c|c|c|}
\hline$(v, k)$ & $q_{k}^{v}$ & $\begin{array}{c}\text { (A) } \\
E\left(u_{\boldsymbol{G}_{1}} \mid c, k, v\right)\end{array}$ & $\begin{array}{c}(B) \\
E\left(u_{\text {chooser }} \mid c, k, v\right)\end{array}$ & $|(A)-(B)|$ & $(A)+(B)$ & $\left((A)+\frac{c+1}{2}\right)\left((B)+\frac{c+1}{2}\right)$ \\
\hline$(1,1)$ & 3 & -1.00 & -4.00 & 3.00 & -5.00 & 0.00 \\
\hline$(2,1)$ & 4 & -1.14 & -3.50 & 2.36 & -4.64 & 1.43 \\
\hline$(2,2)$ & 3 & -1.14 & -3.50 & 2.36 & -4.64 & 1.43 \\
\hline$(1,3)$ & 5 & -4.00 & -1.00 & 3.00 & -5.00 & 0.00 \\
\hline$(3,2)$ & 4 & -1.33 & -3.00 & 1.67 & -4.33 & 2.67 \\
\hline$(3,3)$ & 3 & -1.33 & -3.00 & 1.67 & -4.33 & 2.67 \\
\hline$(4,1)$ & 5 & -4.00 & -1.00 & 3.00 & -5.00 & 0.00 \\
\hline$(4,2)$ & 4 & -1.60 & -2.50 & 0.90 & -4.10 & 3.6 \\
\hline$(4,3)$ & 4 & -1.60 & -2.50 & 0.90 & -4.10 & 3.6 \\
\hline$(4,4)$ & 3 & -1.60 & -2.50 & 0.90 & -4.10 & 3.6 \\
\hline$(5,1)$ & 5 & -4.00 & -1.00 & 3.00 & -5.00 & 0.00 \\
\hline$(5,2)$ & 5 & -4.00 & -1.00 & 3.00 & -5.00 & 0.00 \\
\hline$(5,3)$ & 4 & -2.00 & -2.00 & 0.00 & -4.00 & 4.00 \\
\hline$(5,4)$ & 4 & -2.00 & -2.00 & 0.00 & -4.00 & 4.00 \\
\hline$(5,5)$ & 3 & -2.00 & -2.00 & 0.00 & -4.00 & 4.00 \\
\hline$(6,2)$ & 5 & -4.00 & -1.00 & 3.00 & -5.00 & 4.00 \\
\hline$(6,3)$ & 4 & -2.67 & -1.50 & 1.17 & -4.17 & 3.33 \\
\hline$(6,4)$ & 4 & -2.67 & -1.50 & 1.17 & -4.17 & 3.33 \\
\hline$(6,5)$ & 4 & -2.67 & -1.50 & 1.17 & -4.17 & 3.33 \\
\hline$(6,6)$ & 3 & -2.67 & -1.50 & 1.17 & -4.17 & 3.33 \\
\hline$(7,2)$ & 5 & -4.00 & -1.00 & 3.00 & -5.00 & 0.00 \\
\hline$(7,3)$ & 5 & -4.00 & -1.00 & 3.00 & -5.00 & 0.00 \\
\hline$(7,4)$ & 4 & -4.00 & -1.00 & 3.00 & -5.00 & 0.00 \\
\hline$(7,5)$ & 4 & -4.00 & -1.00 & 3.00 & -5.00 & 0.00 \\
\hline$(7,6)$ & 4 & -4.00 & -1.00 & 3.00 & -5.00 & 0.00 \\
\hline$(7,7)$ & 3 & -4.00 & -1.00 & 3.00 & -5.00 & 0.00 \\
\hline
\end{tabular}




\section{Concluding Remarks}

Rules that contemplate several stages to arrive at a final choice are widely used. Some people are in charge of screening, then others choose among those candidates that were not screened out. The very idea of dividing these tasks may arise from very diverse reasons. One of them, that we consider important but we did not follow here, would be in the line of Condorcet's Jury Theorem for a common values setup: assigning to each agent responsibility for those partial decisions that she is better informed about. Another reason, the one we have focused on in this paper, is to divide the decision power among interested parties whose views may be in conflict. This is in line with Arrowian, tradition, where the preferences of agents are taken as given, and voting rules are methods to arbitrate among them.

In this spirit, we have studied the effective distribution of power that is induced by $v$-rules of $k$ names, a family of two-stage voting procedures that are used to make many appointment decisions around the world, especially in judiciary systems. These rules induce a complete information game that may be described as follows:

1- Each of the proposers casts votes for $v$ candidates;

2- A list of $k$ most voted candidates (after tie-breaking) is provided to the chooser; and,

3- From this list of $k$ names, the chooser selects a single appointee.

We have focused on the strong Nash equilibrium outcome of this game, and discussed the distribution of power that they imply, depending on the choice of the parameters $(v, k)$. Our more specific results are obtained in the context of what we call the polarized opinions model. There, the proposers are partitioned into two groups; all the proposers in each group share the same preferences, and all those in the smaller group also share preferences with the chooser, who is allowed to break eventual ties. Even if simple, this model is able to reflect the potential tension between the majority of the proposers and the chooser, while allowing for the latter to have some allies among those who propose.

The appointment procedure of the members of the State Supreme Court in some US states is an example of institutions where this tension and also these alliances may arise, since one part of the proposers is appointed by the chooser (the governor).

This model admits a relatively simple characterization of the unique strong equilibrium outcome of the associated game. It then allows us to show how a larger gap between the 
parameters $v$ and $k$ can increase the power of the chooser, by allowing her allies to "sneak in" more alternatives in the list, and to proved that the chooser always weakly prefers a smaller $v$ and a larger $k$. We can then also characterize the choice of parameters $(v, k)$ that would get closer to equalize the power of the proposers and the chooser, in terms of the expected rank of the alternatives that they would obtain at equilibrium under a uniform distribution of the potential societies that may be realized. Actually this most egalitarian choice of $(v, k)$ also coincides, in our simple model, with the one that would be recommended through the alternative use of a Nash bargaining or a utilitarian approach.

After this short summary of results, let us now comment on possible extensions. Even within our present framework, we are aware that our normative analysis can be enriched by endowing agents with more complex preferences, considering a wider range of distributions over preference profiles, relaxing the full information assumption and/or considering alternative equilibrium concepts under maybe different specifications of the games within which they interact. But our purpose here was to open a line of work, to provide guidelines for a normative analysis of these widely used rules, and to exhibit the richness and the difficulties involved in following a similar program under alternative assumptions: hence our choice of relatively simple specifications, for utilities, probabilities and equilibrium concept. We think that some of these modeling decisions could be altered without changing the essence of our exercise. Regarding the specification of possible worlds, it is not hard to extend it to cases where the preferences of agents are still based on the ranking of the outcomes but exhibit different degrees of risk aversion. As for the informational assumptions, one could also study easily the polar case where, once a profile is realized, each agent is only informed about her own preference, but remains ignorant about those of the rest. In that case, it becomes natural to assume that agents will behave sincerely, rather than strategically, and the computations carry over in a similar manner.

Finally, let us re-emphasize that, even if widely used, $v$-rules of $k$ names are only one class among many others through which people are eventually appointed. Given the power that comes attached with the possibility to appoint people to offices, we hope that these, along with other rules, can be systematically scrutinized and compared. We would like to think of our work as part of this potential stream of research. 


\section{References}

[1] Alon, N., Fisher, F., Procaccia, A. and Tennenholtz, M., 2011. Sum of us: strategyproof selection from the selectors, in: Proc. 13th Conference on Theoretical Aspects of Rationality and Knowledge 101-110.

[2] Attanasi, G., Corazzini, L. and Passarelli, F., 2010. Voting as a Lottery. No 09-116, TSE Working Papers from Toulouse School of Economics (TSE)

[3] Barberà, S., Sonnenschein, H. and Zhou, L., 1991. Voting by committees. Econometrica $59,595-609$.

[4] Barberà, S., Bossert, W., Pattanaik, P., 2004. Ranking sets of objects. In: Barberà, S., Hammond, P. and Seidl, C.(Eds.) Handbook of Utility Theory, Volume II Extensions. Kluwer Academic Publishers 893-977

[5] Badger, W.W., 1972. Political individualism, positional preferences and optimal decision-rules. In: Niemi, R.G., Weisberg, H.F. (eds.) Probability Methods for Collective Decision Making. Merril Publishing, Columbus, Ohio.

[6] Barberà, S., Jackson, M., 2004. Choosing how to choose: Self-stable majority rules and constitutions. Quarterly Journal of Economics 119, 1011-1048.

[7] Barberà, S., Coelho, D., 2010. On the rule of $k$ names. Games and Economic Behavior $70,44-61$.

[8] Barberà, S., Coelho, D., 2008. How to choose a non-controversial list with $k$ names. Social Choice and Welfare 31, 79-96.

[9] Bernheim, D., Peleg, B. and Whinston, M., 1987. Coalition-proof Nash equilibria I. Concepts. Journal of Economic Theory 42, 1-12.

[10] Brams, S. J., and Merrill, S., 1986. Binding versus final-offer arbitration: A combination is best. Management Science 32, 1346-1355.

[11] de Clippel, G., Eliaz, F. and Knight, B., 2014. On the Selection of Arbitrators. American Economic Review 104, 3434-3458. 
[12] Coelho, D., 2005. Maximin choice of voting rules for committees. Economics of Governance 6, 159-175.

[13] Curtis, R., 1972. Decision rules collective values in constitutional choice. In: Niemi, R.G., Weisberg, H.F. (eds.) Probability Methods for Collective Decision Making. Merril Publishing, Columbus, Ohio.

[14] Ertemel, S., Kutlu, L. and Sanver, R., 2015. Voting games of resolute social choice correspondence. Social Choice and Welfare. Forthcoming.

[15] Gardner, R., 1977. The Borda game. Public Choice 30, 43-50.

[16] Gehrlein W., 1985. The Condorcet criterion and committee selection. Mathematical Social Science 10, 199-209

[17] Holzman, J. and Moulin, H., 2013. Impartial nomination for a prize. Econometrica 81, 173-196.

[18] Kaymak, B. and Sanver, M. R., 2003. Sets of alternatives as Condorcet winners. Social Choice and Welfare 20, 477-494.

[19] Moulin, H., 1982. Voting with proportional veto power. Econometrica 50(1), 145-62.

[20] Moulin, H., 1988. Axioms of Cooperative Decision Making. Cambridge University Press, Cambridge.

[21] Moulin, H. and Peleg, B., 1982. Core of effectivity functions and implementation theory. Journal of Mathematical Economics 10, 115-45.

[22] Mueller, D., 1978. Voting by veto. Journal of Public Economics 10, 57-75.

[23] Peleg, B., 1984. Game Theoretic Analysis of Voting in Committees. Econometric Society Monographs in Pure Theory, Cambridge University Press, Cambridge.

[24] Polborn, M. and Messner, M., 2007. Strong and coalition-proof political equilibria under plurality and runoff rule. International Journal of Game Theory 35, 287-314.

[25] Rae, D., 1969. Decision rules and individual values in constitutional choice. American Political Science Review 63, 40-56. 
[26] Sertel, M. R., and Sanver M. R., 2004. Strong equilibrium outcomes of voting games are the generalized Condorcet winners. Social Choice and Welfare 22, 331-347.

[27] Stevens, C.M., 1966. Is compulsory arbitration compatible with bargaining. Industrial Relations 5, 38-52.

\section{Appendix A}

Proof of Proposition 1. Suppose that candidate $x$ is the outcome of a strong Nash equilibrium of the Constrained Chooser Game. In any strong Nash equilibrium where $x$ is the outcome, the screened set is such that $x$ is the best candidate in this set according to the chooser's preferences. This implies that $x$ is a chooser's $(c-k+1)$-top candidate. To prove that Condition 2 is necessary, take any candidate $y \neq x$ among the chooser's $(c-k+1)$-top candidates, and let $\boldsymbol{Y}$ be any list with $k$ names where $y$ is the chooser's best candidate in $\mathbf{Y}$. Notice that $y$ cannot be considered better than $x$ by any coalition with at least $q_{k}^{v}(\boldsymbol{Y})$ candidates. Otherwise, this coalition could impose $\boldsymbol{Y}$, preventing $x$ from being elected. So, if $y$ is a chooser's $(c-k+1)$-top candidate, then $\#\left\{i \in \mathbf{N} \mid y \succ_{i} x\right\}<q_{k}^{v}(\boldsymbol{Y})$ for any $\boldsymbol{Y} \in \boldsymbol{C}_{k}$ such that $y$ is the chooser's best candidate in $\boldsymbol{Y}$. Now, to justify Condition 3, suppose, by contradiction, that it is not true that $\#\left\{i \in \mathbf{N} \mid y \succ_{i}\right.$ $x\} \geq q_{k}^{v}(y)$. Let $S_{1} \equiv\left\{i \in \mathbf{N} \mid y \succ_{i} x\right\}$, so $\# S_{1} \geq q_{k}^{v}(y)$. Then, the coalition of proposers in $\boldsymbol{C}_{1}$ would be able to impose the inclusion of $y$ in the list (since $\# S_{1} \geq q_{k}^{v}(y)$ ), and the chooser would select it instead of $x$. Hence, if $y$ is the chooser's best candidate, we have that $\#\left\{i \in \mathbf{N} \mid y \succ_{i} x\right\}<q_{k}^{v}(y)$.

Finally, consider Condition 4. Let $y$ be the chooser's best candidate, and assume that it is ranked above $x$ by the tie breaking criterion. Suppose, by contradiction, that it is not true that $\#\left\{i \in \mathbf{N} \mid x \succ_{i} y\right\} \geq q_{k}^{v}$. Hence, at any strategy profile that includes $x$ in the selected list, the coalition $S_{1} \equiv\left\{i \in \mathbf{N} \mid y \succ_{i} x\right\}$ can find a profitable deviation to include $y$, and this becomes the winning candidate. Therefore, $x$ cannot be a strong Nash equilibrium outcome.

Proof of Proposition 2. Let $x$ be the chooser's 1-top candidate. First notice that, since $x$ is a strong Nash equilibrium outcome under a $v^{\prime}$-screening rule for $k^{\prime}$ names, it implies that any strategy profile where all proposers vote for $x$ is a strong Nash equilibrium. Take any strategy profile where all voters vote for $x$, and call it $m^{\prime}$. Given that it is a strong Nash equilibrium, no coalition of voters has a profitable deviation. The voters 
that would wish to avoid the election of $x$ are those that prefer another of the chooser's $\left(c-k^{\prime}+1\right)$-top candidates, rather than $x$ (recall that only the chooser's $\left(c-k^{\prime}+1\right)$-top candidates can be the chooser' best name among the candidates of a set with cardinality $k)$. The only way to avoid the election of $x$ would be to avoid the inclusion of $x$ in the chosen list. Take any chooser's $\left(c-k^{\prime}+1\right)$-top candidate and call it $y$. If all the voters who prefer $y$ to $x$ deviate from $m^{\prime}$ by not voting for $x, x$ would still have enough votes to stay in the $k$ list. Otherwise, the strategy profile where all the voters vote for $x$ would not be a strong Nash equilibrium.

Now we show that $x$ is also a strong Nash equilibrium for any $v$-screening rule for $k$ names where $v \leq v^{\prime}$ and $k \geq k^{\prime}$, by exhibiting a strategy profile that sustains it under the new rule. Take any strategy profile where all voters vote for $x$ and call this strategy profile $m$. Then, $x$ will be one of the $k$ listed names, and it will be the elected candidate, and we need to show that no coalition of voters has a profitable deviation from $m$. Clearly, it is more difficult to find profitable deviations under a $v$-screening rule for $k$ names than under a $v$-screening rule for $k^{\prime}$ names. This is because under a $v$-screening rule for $k$ names any coalition of voters that has incentives to avoid the election of $x$ has less votes to distribute among the $k$ candidates in order to avoid the inclusion of $x$ in the list. Thus, since no coalition has a profitable deviation from $m^{\prime}$, no coalition has one from $m$, either. Therefore, $x$ is a strong Nash equilibrium outcome under $v$-screening rule for $k$ names.

\section{Proof of Proposition 3.}

Consider the case where $\# \boldsymbol{G}_{1} \geq q_{k}^{v}$. Let $x$ be the best alternative of the majoritarian group out of the chooser's $(c-k+1)$-top candidates. Since $\# \boldsymbol{G}_{1} \geq q_{k}^{v}$, by definition of $q_{k}^{v}$, there is a strategy profile that the majoritarian group can adopt and guarantee that $x$ is elected, regardless of the minoritarian group's actions. Notice also that the majoritarian group will not have any incentive in changing this outcome. Therefore, there exists a strategy profile that sustains $x$ as a strong Nash equilibrium outcome.

Now let us show that $x$ is the unique strong Nash equilibrium outcome. Suppose, by contradiction, that there was another strong Nash equilibrium outcome $y \neq x$. By Conditions 1 and 2 of Proposition 1, we have that $x$ and $y$ are among the chooser's $(c-k+1)$-top

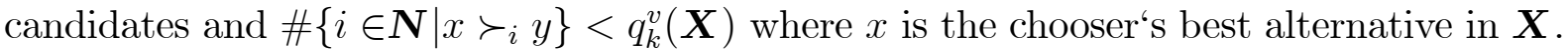
This is a contradiction since $\#\left\{i \in \mathbf{N} \mid x \succ_{i} y\right\} \geqslant \# \mathbf{G}_{1} \geqslant q_{k}^{v} \geqslant q_{k}^{v}(\boldsymbol{X})$.

Consider the case where $q_{k}^{v}>\# \boldsymbol{G}_{1}$. Let $x$ be the chooser's best candidate. Notice that 
$q_{k}^{v}>\# \mathbf{G}_{1}$ implies that $\# \mathbf{G}_{2} \geq q_{k}^{v}(\{x\})$ given that $x$ is the top candidate according to the tie breaking criterion. Consider the strategy profile where every proposer casts a vote for $x$. Then, $x$ will be in the selected list and it will be elected. No group can eliminate $x$ from the selected list by a unilateral deviation, since the two groups have size larger than $q_{v}^{v}(\{x\})$. Since $x$ is $\mathbf{G}_{2}$ 's top candidate, none of the proposers have incentives to jointly deviate from this strategy profile, which therefore sustains $x$ as strong Nash equilibrium outcome.

Now let us prove that $x$ is the unique strong Nash equilibrium outcome. Suppose otherwise, that there exists another strong Nash equilibrium with outcome $y \neq x$. By Condition 3 of Proposition 1, we have that $\#\left\{i \in \boldsymbol{N} \mid x \succ_{i} y\right\}<q_{k}^{v}(\{x\})$. This is a contradiction since $\# \boldsymbol{G}_{1}>\# \mathbf{G}_{2} \geq q_{k}^{v}(\{x\})$.

Proof of Proposition 5. Proposition 5 is a direct consequence of lemmas 1-6.

Lemma 1 Consider any number of candidates $c$ such that $c-\sqrt{2 c+2}+2$ is an integer number and any pair $(v, k)$ such that $\# \boldsymbol{G}_{1} \geq q_{k}^{v}$. If $k=c+2-\sqrt{2 c+2}$ then $E\left(u_{\boldsymbol{G}_{1}}(x) \mid c, k, v\right)=E\left(u_{\text {chooser }}(x) \mid c, k, v\right)$.

Proof of Lemma 1. First notice that for every $(v, k)$ such that $\# \mathbf{G}_{1} \geq q_{k}^{v}$, by Proposition 4 , we have that the product of $E\left(u_{\boldsymbol{G}_{1}}(x) \mid c, k, v\right)$ and $E\left(u_{\text {chooser }}(x) \mid c, k, v\right)$ is equal to $\frac{c+1}{2}$.

Take any $k^{*} \in\{1, \ldots, c\}$ and $v^{*} \in\{1, \ldots, k\}$ such that $\# \boldsymbol{G}_{1} \geq q_{k}^{v}$ and $E\left(u_{G 1}(x) \mid c, k^{*}, v^{*}\right)=$ $E\left(u_{\text {chooser }}(x) \mid c, k^{*}, v^{*}\right)$. Thus,

$E\left(u_{\text {chooser }}(x) \mid c, k^{*}, v^{*}\right)^{2}=\frac{c+1}{2}$. It implies that:

$E\left(u_{\text {chooser }}(x) \mid c, k^{*}, v^{*}\right)=\frac{c-k^{*}+2}{2}=\sqrt[2]{\frac{c+1}{2}}$

Therefore, $k^{*}=c+2-\sqrt[2]{2 c+2}$.

Lemma 2 A pair $(v, k)$, such that $\# \mathbf{G}_{1} \geq q_{k}^{v}$, maximizes $E\left(u_{\boldsymbol{G}_{1}}(x) \mid c, k, v\right)+E\left(u_{\text {chooser }}(x) \mid c, k, v\right)$ if and only if it minimizes $\left.\mid E\left(u_{\boldsymbol{G}_{1}}(x) \mid c, k, v\right)-E\left(u_{\text {chooser }}(x) \mid c, k, v\right)\right) \mid$.

Proof of Lemma 2. First notice that for every $(v, k)$ such that $\# \mathbf{G}_{1} \geq q_{k}^{v}$, by Proposition 4, we have that:

$E\left(u_{G_{1}}(x) \mid c, k, v\right) E\left(u_{\text {chooser }}(x) \mid c, k, v\right)=\frac{c+1}{2}$

The equality above implies that

$\left(E\left(u_{\boldsymbol{G}_{1}}(x) \mid c, k, v\right)+E\left(u_{\text {chooser }}(x) \mid c, k, v\right)\right)^{2}=E\left(u_{\boldsymbol{G}_{1}}(x) \mid c, k, v\right)^{2}+E\left(u_{\text {chooser }}(x) \mid c, k, v\right)^{2}+$ 
$(c+1)$

The expression above implies that, since that $E\left(u_{\boldsymbol{G}_{1}}(x) \mid c, k, v\right)+E\left(u_{\text {chooser }}(x) \mid c, k, v\right)<0$, a $k \in\{1, \ldots c\}$ maximizes $E\left(u_{G 1}(x) \mid c, k\right)+E\left(u_{c h o o s e r}(x) \mid c, k, v\right)$ if and only if it minimizes $E\left(u_{G_{1}}(x) \mid c, k, v\right)^{2}+E\left(u_{c h o o s e r}(x) \mid c, k, v\right)^{2}$.

Notice also that:

$\left(E\left(u_{\boldsymbol{G}_{1}}(x) \mid c, k, v\right)-E\left(u_{\text {chooser }}(x) \mid c, k, v\right)\right)^{2}=E\left(u_{\boldsymbol{G}_{1}}(x) \mid c, k, v\right)^{2}+E\left(u_{\text {chooser }}(x) \mid c, k, v\right)^{2}-$ $(c+1)$.

The expression above implies that a $k \in\{1, \ldots c\}$ maximizes $E\left(u_{G_{1}}(x) \mid c, k, v\right)^{2}+E\left(u_{c h o o s e r}(x) \mid c, k, v\right)^{2}$ if and only if it maximizes $\left(E\left(u_{\boldsymbol{G}_{1}}(x) \mid c, k, v\right)-E\left(u_{\text {chooser }}(x) \mid c, k, v\right)\right)^{2}$.

Therefore, a $k \in\{1, \ldots c\}$ maximizes $E\left(u_{G_{1}}(x) \mid c, k, v\right)+E\left(u_{\text {chooser }}(x) \mid c, k, v\right)$ if and only if minimizes $\left.\mid E\left(u_{G 1}(x) \mid c, k, v\right)-E\left(u_{c h o o s e r}(x) \mid c, k, v\right)\right) \mid$.

Lemma 3 A pair $(v, k)$, such that $\# \boldsymbol{G}_{1} \geq q_{k}^{v}$, maximizes $E\left(u_{\boldsymbol{G}_{1}}(x) \mid c, k, v\right)+E\left(u_{c h o o s e r}\left(r_{c}\right) \mid c, k, v\right)$ if and only if it also maximizes $\left(E\left(u_{G_{1}}(x) \mid c, k, v\right)-d\right)\left(E\left(u_{\text {chooser }}(x) \mid c, k, v\right)-d\right)$ where $d<0$.

Proof of Lemma 3. First notice that for every $(v, k)$ such that $\# \boldsymbol{G}_{1} \geq q_{k}^{v}$, by Proposition 4, we have that:

$E\left(u_{\boldsymbol{G}_{1}}(x) \mid c, k, v\right) E\left(u_{\text {chooser }}(x) \mid c, k, v\right)=\frac{c+1}{2}$.

Thus, $\left(E\left(u_{\boldsymbol{G}_{1}}(x) \mid c, k, v\right)-d\right)\left(E\left(u_{\text {chooser }}(x) \mid c, k, v\right)-d\right)=\frac{c+1}{2}+d^{2}-d\left(E\left(u_{\boldsymbol{G}_{1}}(x) \mid c, k, v\right)+\right.$ $\left.E\left(u_{\text {chooser }}(x) \mid c, k, v\right)\right)$

Since $d<0$, the expression above implies that $k$ maximizes $E\left(u_{\boldsymbol{G}_{1}}(x) \mid c, k, v\right)+E\left(u_{\text {chooser }}(x) \mid c, k, v\right)$ if and only if it maximizes $\left(E\left(u_{\boldsymbol{G}_{1}}(x) \mid c, k, v\right)-d\right)\left(E\left(u_{\text {chooser }}(x) \mid c, k, v\right)-d\right)$.

Lemma 4 Consider any number of candidates $c,\left(v^{\prime}, k\right)$ and $\left(v^{\prime}, k-1\right)$ such that $k \geq$ $2, \# \boldsymbol{G}_{1} \geq q_{k}^{v^{\prime}}$ and $\# \boldsymbol{G}_{1} \geq q_{k-1}^{v^{\prime}}$ :

1)If $k<c+\frac{5}{2}-\sqrt{2 c+\frac{9}{4}}$ then $E\left(u_{G 1}(x) \mid c, k, v^{\prime}\right)+E\left(u_{\text {chooser }}(x) \mid c, k, v\right)>E\left(u_{G 1}(x) \mid c, k-\right.$ $\left.1, v^{\prime}\right)+E\left(u_{\text {chooser }}(x) \mid c, k-1, v^{\prime}\right)$;

2)If $k=c+\frac{5}{2}-\sqrt{2 c+\frac{9}{4}}$ then $E\left(u_{G 1}(x) \mid c, k, v^{\prime}\right)+E\left(u_{c h o o s e r}(x) \mid c, k, v^{\prime}\right)=E\left(u_{G 1}(x) \mid c, k-\right.$ $\left.1, v^{\prime}\right)+E\left(u_{\text {chooser }}(x) \mid c, k-1, v^{\prime}\right)$;

3)If $k>c+\frac{5}{2}-\sqrt{2 c+\frac{9}{4}}$ then $E\left(u_{G 1}(x) \mid c, k, v^{\prime}\right)+E\left(u_{\text {chooser }}(x) \mid c, k, v^{\prime}\right)<E\left(u_{G 1}(x) \mid c, k-\right.$ $\left.1, v^{\prime}\right)+E\left(u_{\text {chooser }}(x) \mid c, k-1, v^{\prime}\right)$ 
Proof of Lemma 4. Since $\left(v^{\prime}, k\right)$ and $\left(v^{\prime}, k-1\right)$ such that $k \geq 2, \# \mathbf{G}_{1} \geq q_{k}^{v^{\prime}}$ and $\# \mathbf{G}_{1} \geq q_{k-1}^{v^{\prime}}$, by Proposition 4 , we have the following equality:

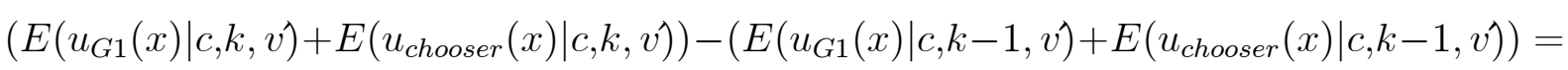
$\frac{c+1}{(c-k+2)(c-k+3)}-\frac{1}{2}$.

Notice $\frac{c+1}{(c-k+2)(c-k+3)}$ is decreasing with $k$ and $\frac{c+1}{(c-k+2)(c-k+3)}=\frac{1}{2}$ when $k=c+\frac{5}{2}-\sqrt{2 c+\frac{9}{4}}$. Let $\boldsymbol{P}(k)=\frac{c+1}{(c-k+2)(c-k+3)}-\frac{1}{2}$ and $k^{*}=c+\frac{5}{2}-\sqrt{2 c+\frac{9}{4}}$. Thus, $\boldsymbol{P}\left(k^{*}\right)=0, \boldsymbol{P}(k)>0$ for any $k<k^{*}$ and $\boldsymbol{P}(k)<0$ for any $k>k^{*}$.

Lemma 5 If $c-\sqrt{2 c+2}+2$ is an integer number then it is equal to $\left\lfloor c+\frac{5}{2}-\sqrt{2 c+\frac{9}{4}}\right\rfloor$.

Proof of Lemma 5. Let $z=c+\frac{5}{2}-\sqrt{2 c+\frac{9}{4}}$ and $y=c-\sqrt{2 c+2}+2$. Notice that $z-y=\sqrt{2 c+2}+\frac{1}{2}-\sqrt{2 c+2+\frac{1}{4}}$. Thus, $1>z-y>0$ for every $c>0$. Therefore if $c-\sqrt{2 c+2}+2$ is an integer number we have that $\left\lfloor c+\frac{5}{2}-\sqrt{2 c+\frac{9}{4}}\right\rfloor=c-\sqrt{2 c+2}+2$.

Lemma 6 Let $z=c+\frac{5}{2}-\sqrt{2 c+\frac{9}{4}}$.

a) For any $c \epsilon\{3,4,6,7,8\}$, $z$ is not a integer and $\lfloor z\rfloor \geq \frac{c+1}{2}$;

b) If $c=5$ then $z$ is a integer and $z-1 \geq \frac{c+1}{2}$;

c) $(z-1)-(c+1) / 2 \geq 0$ for any $c \geq 9$.

Proof of Lemma 6. The itens a and b can be verified in the table below that shows the values of $z$ and $\frac{c+1}{2}$ for every $c \epsilon\{3,4,5,6,7,8\}$.

\begin{tabular}{ccc}
\hline$c$ & $z$ & $(c+1) / 2$ \\
\hline 3 & 2.63 & 2 \\
4 & 3.30 & 2.5 \\
5 & 4 & 3 \\
6 & 4.73 & 3.5 \\
7 & 5.47 & 4 \\
8 & 6.23 & 4.5 \\
\hline
\end{tabular}

For any $c>8$, let us prove that $(z-1)-(c+1) / 2 \geq 0$.

$(z-1)-(c+1) / 2=c+\frac{3}{2}-\sqrt{2 c+\frac{9}{4}}-\frac{c+1}{2}=\frac{1}{2} c+1-\sqrt{2 c+\frac{9}{4}}$
$=\frac{2 c+4}{4}-\sqrt{\frac{8 c+9}{4}}>\frac{2 c+4}{4}-\sqrt{\frac{9 c+9}{4}}=\frac{\sqrt{c+2} \sqrt{c+2}}{2}-3 \frac{\sqrt{c+1}}{2}>\frac{\sqrt{c+1}}{2}(\sqrt{c+2}-3)=$
$=\frac{\sqrt{c+1}}{2}(\sqrt{c+2}-3)>0$. 


\section{Appendix B}

Proposition 1 helps us to locate equilibria and provides a first step toward their characterization, when they exist. But knowing the necessary conditions alone is already of great help. We illustrate this point though an example.

Example 5 Let $\boldsymbol{C}=\{c 1, c 2, c 3, c 4, c 5\}$ and let $\mathbf{N}=\{1,2,3\}$. Suppose that each proposer votes for one candidate and the three most voted candidates form the list, with a tie breaking rule when needed: $c 2 \succ c 1 \succ c 5 \succ c 4 \succ c 3$. The preferences of the chooser and the committee members are as follows:

\section{Preference Profile}

Proposer 1 Proposer 2 Proposer 3 Chooser

$\begin{array}{llll}c 5 & c 5 & c 5 & c 1 \\ c 4 & c 4 & c 4 & c 2 \\ c 3 & c 3 & c 2 & c 3 \\ c 1 & c 1 & c 1 & c 4 \\ c 2 & c 2 & c 3 & c 5\end{array}$

Notice that, we have that $q_{k}^{v}(\{x\})=1$ for any $x \in \boldsymbol{C}$ and $q_{k}^{v}(\boldsymbol{X})=3$ for any $\boldsymbol{X} \in \boldsymbol{C}_{k}$.

The first step in describing the equilibrium outcomes is to identify those candidates that satisfy the three necessary conditions established in Proposition 1.

Inspecting the preference profile above, we have that:

1. Condition 1: $\{c 1, c 2, c 3\}$.

2. Condition 2: $\{c 1, c 2, c 3, c 4, c 5\}$.

3. Condition 3: $\{c 1, c 4, c 5\}$.

4. Condition 4: $\{c 1, c 2, c 4, c 5\}$.

So, only candidate $c 1$ that satisfies all four conditions. Now we have to check whether there is a strategy profile that sustains candidate $c 1$ as a strong Nash equilibrium candidate. The following strategy profile sustains $\mathrm{c} 1$ as a strong Nash equilibrium outcome: Proposer 1 votes for 1 , Proposer 2 votes for $c 4$ and Proposer 3 votes for $c 2$.

In the preceding example, the choice of candidates satisfying the necessary conditions could in fact be sustained with an appropriate set of strong equilibrium strategies. But this does not need to be the case. In fact, there may be candidates that satisfy the 
necessary conditions and yet cannot be the outcome of any equilibrium. Worse than that, equilibria may not exist even if some candidates meet the necessary conditions, as shown by our next example.

Example 6 Let $\boldsymbol{C}=\{c 1, c 2, c 3, c 4, c 5, c 6\}$ and let $\mathbf{N}=\{1,2,3\}$. The proposers use the rule of 4 names, $(v=1, k=4)$, with the following tie breaking rule when needed: $c 6 \succ c 5 \succ c 4 \succ c 3 \succ c 2 \succ c 1$. The preferences of the chooser and the committee members are as follows:

\begin{tabular}{cccccc}
\multicolumn{7}{c}{ Preference Profile } \\
Proposer 1 & Proposer 2 & Proposer 3 & Proposer 4 & Proposer 5 & Chooser \\
$c 5$ & $c 5$ & $c 5$ & $c 5$ & $c 1$ & $c 1$ \\
$c 6$ & $c 6$ & $c 6$ & $c 6$ & $c 3$ & $c 2$ \\
$c 4$ & $c 4$ & $c 4$ & $c 4$ & $c 2$ & $c 3$ \\
$c 2$ & $c 2$ & $c 2$ & $c 2$ & $c 4$ & $c 4$ \\
$c 3$ & $c 3$ & $c 3$ & $c 3$ & $c 6$ & $c 5$ \\
$c 1$ & $c 1$ & $c 1$ & $c 1$ & $c 5$ & $c 6$
\end{tabular}

First, notice that $q_{k}^{v}(\{x\})=1$ for any $x \in\{c 3, c 4, c 5, c 6\}, q_{k}^{v}(\{x\})=2$ for any $x \in\{c 1, c 2\}$ and $q_{k}^{v}(\boldsymbol{X})=5$ for any $\boldsymbol{X} \in \boldsymbol{C}_{k} \backslash\{c 3, c 4, c 5, c 6\}$ and $q_{k}^{v}(\{c 3, c 4, c 5, c 6\})=4$. The first step in describing the equilibrium outcomes is to identify those candidates that satisfy the three necessary conditions established in Proposition 1.

Inspecting the preference profile above, we have that:

1. Condition 1: $\{c 1, c 2, c 3\}$.

2. Condition 2: $\{c 2, c 3, c 4, c 5, c 6\}$.

3. Condition 3: $\{c 1, c 2, c 3, c 4, c 5, c 6\}$.

4. Condition 4: $\{c 1, c 2, c 3, c 4, c 5, c 6\}$.

So, only candidates $c 2$ and $c 3$ satisfy all four conditions. However, there exists no strategy profile that can sustain them as a strong Nash equilibrium outcome of the Constrained Chooser Game. The reason is simple: for any strategy profile that would lead the election of c2, Proposer 5 would have a profitable deviation by casting a vote for $c 3$ or $c 1$ and for any strategy profile that would lead the election of 33 , proposers 1,2,3 and 4 have a profitable deviation by casting votes for $c 2$. 